\title{
The effect of study modality on false recognition
}

\author{
RebeKah E. Smith and R. Reed Hunt \\ University of Texas, San Antonio, Texas \\ AND \\ M. Patrick Gallagher \\ Wake Forest University, Winston-Salem, North Carolina
}

\begin{abstract}
A number of previous studies have shown that false recognition of critical items in the Deese/RoedigerMcDermott paradigm is reduced when study items are presented visually rather than auditorily; however, this effect has not been uniformly demonstrated. We investigated three potential boundary conditions of the effect of study modality in false recognition. Experiments 1 and 2 showed no reduction in false recognition following visual study presentation when the yes-no recognition test was not preceded by a recall test. Experiment 3 showed that visual study presentation can reduce false recognition without a preceding recall test, if the recognition test uses remember-know instructions. The order of the recognition test items did not influence the effect of visual study presentation on false recognition in Experiment 1. In general, the data imply that distinctive processing at study can reduce false memory in recognition if the test demands draw attention to the dimension of distinctive processing.
\end{abstract}

Memory accuracy requires correct identification both of events that did occur and of events that did not occur. Failure to reject events that did not occur yields false memories, a phenomenon that has received renewed attention with Roediger and McDermott's (1995) resuscitation of Deese's (1959) paradigm. In the Deese/RoedigerMcDermott (DRM) paradigm, participants study lists of words, such as SOUR, CANDY, HEART, and so on, that are all highly associated with a critical nonstudied item, such as SWEET. On subsequent recall and recognition tests, participants will frequently falsely recall or falsely recognize the critical item as having been part of the study list (e.g., Payne, Elie, Blackwell, \& Neuschatz, 1996; Read, 1996; Roediger \& McDermott, 1995). Levels of false recall and recognition are often as high as levels of correct recall and recognition. Thus, the DRM paradigm provides a reliable way to evoke false memories.

Smith and Hunt (1998) discovered that the probability of falsely recalling or falsely recognizing the critical items in the DRM paradigm is significantly lower following visual study presentation than following auditory study presentation. The effect of study modality has now been demonstrated using the DRM paradigm on written recall tests in 10 different experiments (Cleary \& Greene, 2002; Gallo, McDermott, Percer, \& Roediger, 2001, Experiment 1; Kellogg, 2001; Smith \& Hunt, 1998; Smith, Lozito, \& Bayen, 2005) and on visual recognition tests in 8 experiments (Cleary \& Greene, 2002; Gallo et al., 2001; Gallo \& Roediger, 2003; Pierce, Gallo, Weiss, \& Schacter, 2005, Experiment 1; Smith \& Hunt, 1998). Although the reduc- tion of false memories following visual study presentation has been replicated in a number of different studies from different laboratories, the size of the effect in recognition memory varies across studies, and in some cases visual study does not reduce false recognition relative to auditory study (Kellogg, 2001; Maylor \& Mo, 1999; McKone \& Murphy, 2000; Pierce et al., 2005, Experiment 2). The purpose of the present study was to investigate potential boundary conditions for the effect of study presentation modality on false recognition in the DRM paradigm.

In service of this goal, we examined the literature to identify factors that may influence whether visual study presentation reduces false recognition. One factor has already been identified in the literature-namely, the modality of the final memory test. In two experiments conducted by Gallo et al. (2001, Experiments 2 and 3), visual study presentation did not reduce false recognition when an auditory recognition test was used; thus, the modality of the recognition test does influence the effect of study modality (see also Kellogg, 2001, for parallel findings in false recall). Kellogg suggested that when a written memory test is used, the match between study and test modalities in the visual study condition may underlie the reduction in false memory relative to the case in which there is a mismatch between study and test (auditory study followed by visual test). However, if this explanation were correct, we would expect to see a reduction of false memories on an auditory recognition test following auditory study relative to an auditory test following visual study, and this is not the case (Gallo et al., 2001, Experiments 1 and 2).

R. E. Smith, rebekah.smith@utsa.edu 
In this study, we focused primarily on two additional factors: the presence or absence of a recall test prior to the recognition test and the use of remember-know instructions on a recognition test. (Discussion of a third factor, the order of the recognition test items, can be found in Experiment 1 . This factor had no effect on whether study modality reduced false recognition; therefore, we focused the introduction on the first two factors.) This is certainly not an exhaustive list, and factors such as presentation rate, the number of associate lists presented, or the number of associates presented in each list might also influence the expression of the effect of study modality. However, these two factors stood out as the most likely candidates that moderate the influence of visual study presentation on false recognition. Furthermore, the potential effects of these two factors can be explained by a single theoretical process, and the experiments presented here provide an evaluation of that idea.

The theoretical approach is based on Smith and Hunt's (1998) explanation of the reduction in false memories following visual study, an explanation that draws on the concepts of both relational and item-specific processing. Relational processing refers to the processing of dimensions shared by all of the items, and item-specific processing refers to the processing of information unique to individual items (Hunt, 2003; Hunt \& Einstein, 1981; Hunt \& McDaniel, 1993; Hunt \& Worthen, 2006). One source of relational information in the DRM paradigm is the semantic relationship between the critical and the studied items. This relational information may lead to the critical item's coming to mind during study, during the memory test, or during both study and test. The relational information would also encourage endorsement of the critical item in recall and recognition. A second potential source of relational processing for the studied items is the modality of study presentation. Although study modality can serve as a source of shared relational information for the studied items, this relational information does not extend to the nonstudied critical items. ${ }^{1}$ The studied items are perceptually processed, whereas the critical item is not. Thus, the modality of study presentation provides a dimension along which the critical lures can be differentiated from the studied items.

A second assumption in Smith and Hunt's (1998) explanation of the reduction in false memories following visual study presentation is that visual processing is less like the conceptual activation (thought) that brings the critical item to mind than is auditory processing. This assumption has some indirect support in the reality-monitoring literature (Johnson, Nolde, \& De Leonardis, 1996) and in the false memory literature (Hicks \& Marsh, 2001). If visual processing is less like thought than is auditory processing, it should be easier to discriminate items visually presented at study from items that may have come to mind but were not perceived than it would be to discriminate between auditorily presented study items and items that come to mind that were not perceived.

With respect to false recall, we assumed that free recall tests encourage participants to consider the quality of their memory for a given potential response and to compare that potential response with other responses. This is so because the free recall test does not require that every response that comes to mind be output, and indeed, most of the experiments showing a reduction in false recall following visual study presentation include instructions not to guess on the recall test (e.g., Gallo et al., 2001; Kellogg, 2001; Smith \& Hunt, 1998; Smith et al., 2005; not specified in Cleary \& Greene, 2002). Thus, the monitoring engaged by a recall test can encourage discrimination of studied items and nonpresented items. The discrimination of studied and nonstudied items will be facilitated by visual study presentation in free recall.

In recognition, however, the critical items will always come to mind because they are present on the test and must be subjected to a recognition decision. In order to decide that a critical item was not part of the study list, the dimension along which the studied and critical items can be distinguished must be processed. In other words, the test must encourage processing of the quality of our memory for each item, including whether that memory includes perceptual information. In a yes-no recognition task, the test context may not encourage thorough processing of the quality of the memory. In this case, the visual study presentation will not be effective in reducing false alarms. However, if a recall test precedes recognition, the evaluative processing that we suggest occurs in recall may transfer to the recognition test. ${ }^{2}$ If so, the yes-no recognition test that follows a recall test may benefit from the processing of the perceptual aspects of the memory for studied items and the lack thereof for the critical items encouraged by the recall test. This prediction was tested in Experiments 1 and 2.

The preceding theoretical approach can explain the smaller reductions in false memories following visual study presentation in Gallo et al. (2001, Experiments 2 and 3) and Gallo and Roediger (2003). The approach can also reconcile the inconsistency between our failure to find a reduction in false recognition following visual study in the absence of a prior recall and the small but reliable effect reported by Gallo et al. and by Gallo and Roediger (2003) in the absence of recall. In the first experiment of Gallo et al., the effect of prior recall on recognition was based on a within-subjects manipulation of prior recall. The trials involving a recall test could encourage processing of the quality of potential responses. This processing then could transfer to the recognition test on that trial. Moreover, in the within-subjects design, this comparative processing could also be applied to the recognition test on trials that did not involve a recall test. The other experiments of Gallo et al. and Gallo and Roediger (2003) had no prior recall. What they did have that our first two experiments lacked was a recognition test that required either a remember-know judgment (see also Pierce et al., 2005) or a source judgment. These judgments may require or encourage additional processing of possible recognition test responses of the kind we suggest occurs in standard written free recall. If so, one might expect a reduction in false recognition following visual study, albeit a potentially smaller reduction than would normally be seen with a prior recall. We tested this prediction in Experiment 3. 


\section{EXPERIMENT 1}

Cleary and Greene (2002) and Smith and Hunt (1998) employed a recall test prior to a recognition test in the DRM paradigm and reported significant reductions in false recognition following visual, as compared with auditory, study. Significant but smaller effects were obtained by Gallo et al. (2001) and Gallo and Roediger (2003). With the exception of the first experiment in Gallo et al., the experiments in Gallo et al. and Gallo and Roediger (2003) did not include a recall test. Likewise, Maylor and Mo (1999) compared visual and auditory presentation on recognition memory with no prior recall tests, and their results are the only such direct comparison that yielded at least a numerical reduction in false recognition following auditory study, relative to following visual study. To determine whether the presence of a prior recall test influences the extent to which study presentation reduces false recognition, we manipulated the presence/absence of the recall test in a between-subjects design in Experiment 1.

Although this experiment was conducted for the primary purpose of investigating the potential effects of the presence or absence of a recall test prior to the recognition test, a second factor was also investigated. In Experiment 1 , we included a manipulation of the structure of the recognition test. In the original study by Smith and Hunt (1998), the recognition test was modeled after that used by Roediger and McDermott (1995, Experiment 1). For each list of associates, the recognition test included the critical item, two weakly related lures, two unrelated lures, and two studied items. On the test, the items associated with a given critical item were presented in a block that began with the highest associate and ended with the critical item. Studies showing smaller reductions in false recognition following visual study have presented recognition test items in a random order (Gallo et al., 2001; Gallo \& Roediger, 2003), rather than blocked by associate list (Cleary \& Greene, 2002; Smith \& Hunt, 1998). Maylor and Mo (1999) also used randomly ordered recognition tests. To determine whether random presentation of the items on the recognition test reduces or eliminates the reduction in false recognition associated with visual study presentation, test presentation (blocked vs. random) was manipulated orthogonally to the presence or absence of a recall test.

\section{Method}

\section{Participants}

Participants in all experiments were students enrolled in introductory psychology courses who volunteered for the study in exchange for credit toward a course requirement. Participants were native English speakers.

\section{Design}

Half of the 232 participants were administered a recall test following each list of associates, and half attempted to solve puzzles after each list. Within each of these groups, half of the participants saw the study list words, and the other half heard the study list words. Within each modality group, participants were randomly assigned to either the ordered recognition test condition or the random recognition test condition. Thus, the experiment involved a 2 (recall condition: recall vs. puzzle) $\times 2$ (modality: visual vs. auditory) $\times$
2 (recognition test type: ordered vs. random) design. There were 29 participants in each of the eight conditions.

\section{Materials}

The six critical items (CHAIR, MOUNTAIN, NEEDLE, ROUGH, SWEET, SLEEP) that were most likely to be falsely remembered in the Roediger and McDermott (1995) study were selected for the present experiment. The study lists consisted of the 12 highest associates of each of the six critical items, presented in order from the highest associate to the 12th associate. Study items were presented via computers. In the visual condition, the words were presented in the center of the computer screen, displayed in black lowercase letters in an 18-point font. In the auditory condition, participants heard the words, recorded in WAV files, through headphones connected to each individual computer.

The recognition test consisted of 42 words, 7 items for each set of associates. The 7 items consisted of 2 studied items (the highest associate and an additional studied item randomly selected from Positions $2-6$ on the associate list), the critical item lure, 2 weakly related lures (Positions 14 and 15 on the associate list), and 2 unrelated lures (Positions 14 and 15 of six nonstudied associate lists). In the ordered recognition test condition, the items were presented in a blocked fashion, starting with the highest associate and ending with the critical item. The other 5 items (the 2 nd studied item, the 2 weakly related lures, and the 2 unrelated lures) were presented randomly between the highest associate and the critical item, with the restriction that no 2 items of the same class (studied, related lures, unrelated lures) occurred in sequence. These materials match those used in previous false memory studies (e.g., Smith \& Hunt, 1998). In the random recognition test condition, all items were presented in a random order, with the restriction that no 2 items from a given associate list were presented in sequence.

\section{Procedure}

After receiving standard intentional memory instructions, participants heard or saw each set of 12 associates at a rate of $1.5 \mathrm{sec}$ per word. Following each set of associates, participants in the recall group completed a recall test for that particular list. Participants were given a booklet with six blank pages and were instructed to recall as many words as they could from the previous list, but not to guess randomly. Following each 2.5 -min recall period, participants turned to a blank page in the recall booklet before seeing or hearing the next set of associates. The recall booklet was collected after the last recall period and was, therefore, not present when participants completed the recognition test.

In the puzzle group, participants worked on a nonverbal puzzle during the 2.5 -min period following each associate list. The solitaire puzzle involved a small game board with 33 squares arranged in a "+" pattern. At the start of the game, each square, except for the very center square, contained a game piece. The goal of the puzzle was to jump one game piece over another and into an empty space (i.e., the first jump had to be to the center square). The piece that was jumped was removed from the board. The goal of the puzzle was to finish with a single piece on the board in the center location. The participants could start the game over if they removed all of the pieces without solving the puzzle. This puzzle task was selected because of its engaging nature. All participants appeared to work on the task continuously during the delay, and no participant successfully solved the puzzle in the time allotted.

After the last recall test or puzzle period, the participants completed a yes-no recognition test. Test words were presented individually on the monitor, and the participants were instructed to respond by pressing the "Y" key if the word had been in the study list or the "N" key if the word had not been in the study list. The test was self-paced, and the participants were instructed not to guess.

\section{Results and Discussion}

An alpha level of .05 was used in tests of statistical significance across all experiments. Effect sizes will be 
reported for all statistically significant comparisons in the form of partial eta squared $\left(\eta_{\mathrm{p}}^{2}\right)$.

\section{Recall}

In the analysis of recall test performance, the omnibus test produced main effects of item type (correct studied item vs. falsely recalled critical item) $[F(1,114)=135.27$, $\left.M S_{\mathrm{e}}=.03, \eta_{\mathrm{p}}^{2}=.54\right]$ and study modality $[F(1,114)=$ $\left.12.46, M S_{\mathrm{e}}=.03, \eta_{\mathrm{p}}^{2}=.10\right]$, which were qualified by a significant interaction of the two variables $[F(1,114)=$ $\left.12.12, M S_{\mathrm{e}}=.03, \eta_{\mathrm{p}}^{2}=.10\right]$. We investigated the interaction with separate analyses for each item type.

Correct recall. As in previous studies (e.g., Gallo et al., 2001; Smith \& Hunt, 1998), presentation modality at study did not significantly affect correct recall $(F<1)$. In both the auditory and visual conditions, the mean proportion of studied items correctly recalled was .67 (SEM = .01 in both cases).

False recall. The proportion of the six critical items that were incorrectly produced on the recall tests for the 116 participants in the recall group is shown in Table 1 as a function of modality. False recall was significantly lower following visual presentation $(M=.32, S E M=$ $.03)$ than it was following auditory presentation $(M=.48$, $S E M=.03)\left[F(1,114)=14.52, M S_{\mathrm{e}}=.05, \eta_{\mathrm{p}}^{2}=.11\right]$. Thus, the reduction in critical item false recall following visual study relative to auditory study that has been seen in previous studies (Cleary \& Greene, 2002; Gallo et al., 2001, Experiment 1; Kellogg, 2001; Smith \& Hunt, 1998; Smith et al., 2005) was replicated in this experiment.

\section{Recognition}

We conducted a mixed ANOVA with the repeated measure of item type and the between-subjects variables of study modality, recall condition (recall test vs. no recall test prior to the recognition test), and recognition test type (ordered vs. random). The main effect of item type $\left[F(1,224)=33.98, \eta_{\mathrm{p}}^{2}=.13\right]$ was qualified by significant interactions with study modality $\left[F(1,224)=19.00, \eta_{\mathrm{p}}^{2}=\right.$ $.08]$ and recall condition $\left[F(1,224)=16.35, \eta_{\mathrm{p}}^{2}=.07\right]$ $\left(M S_{\mathrm{e}}=.02\right.$ in all cases $)$. The main effect of study modality approached but did not reach significance $[F(1,224)=$ $\left.3.90, M S_{\mathrm{e}}=.003, p<.06, \eta_{\mathrm{p}}^{2}=.02\right]$. The main effect of recall condition was not significant $[F(1,224)=1.29$, $\left.M S_{\mathrm{e}}=.03\right]$. However, there was a significant interaction between study modality and recall condition $[F(1,224)=$
12.16, $\left.M S_{\mathrm{e}}=.03, \eta_{\mathrm{p}}^{2}=.05\right]$. The three-way interaction of study modality, recall condition, and item type did not reach significance $\left[F(1,224)=2.65, M S_{\mathrm{e}}=.02, p>.10\right]$. Recognition test type did not significantly affect recognition test performance and did not interact with any other variable (all $F_{\mathrm{S}}<1$ ); thus, we collapsed over this variable in the remaining analyses. We investigated the significant interactions of item type with study modality and recall condition with separate analyses for each item type.

Correct recognition. The proportion of studied items correctly identified was affected significantly by the prior presence of a recall test $\left[F(1,228)=24.26, M S_{\mathrm{e}}=.01\right.$, $\left.\eta_{\mathrm{p}}^{2}=.10\right]$, and there was a trend $(p<.10)$ for an effect of study modality $\left[F(1,228)=2.91, M S_{\mathrm{e}}=.01, \eta_{\mathrm{p}}^{2}=\right.$ $.01]$. The interaction of modality and recall condition $\left[F(1,228)=6.07, M S_{\mathrm{e}}=.01, \eta_{\mathrm{p}}^{2}=.03\right]$ was significant. We evaluated the effects of modality on correct recognition for each recall condition separately. In the case of the recall group, there was no effect of modality $(F<1)$. Correct recognition following visual study and recall tests was $.92(S E M=.01)$, as compared with .91 $(S E M=.01)$ following auditory study and recall tests. In contrast, there was a significant effect of modality on correct recognition in the puzzle group $\left[F(1,114)=6.58, M S_{\mathrm{e}}=.02\right.$, $\left.\eta_{\mathrm{p}}^{2}=.06\right]$. Correct recognition in the visual condition was $.87(S E M=.02)$, as compared with $.81(S E M=.02)$ in the auditory condition. This effect was not predicted, nor was it replicated in Experiment 2 or 3 . The existing literature shows mixed findings regarding the effects of study modality on correct recognition. Some experiments have shown no effect of study modality on correct recognition (Gallo et al., 2001, Experiment 1; Gallo \& Roediger, 2003; Pierce et al., 2005; Smith \& Hunt, 1998). Others have shown a benefit of visual study modality over auditory study modality on correct recognition (Cleary \& Greene, 2002; Gallo et al., 2001, Experiments 2 and 3). Finally, Maylor and Mo (1999) reported correct recognition that was at least numerically higher following auditory study than following visual study (the specific analysis was not reported). Thus, the effects of study modality on correct recognition remain unclear.

False recognition of critical items. The proportion of critical item false alarms is shown in Table 1 as a function of modality and group. The main effect of recall condition (recall vs. puzzle) was not significant $[F(1,228)=$ $\left.1.81, M S_{\mathrm{e}}=.04\right]$, and the main effect of study modality

Table 1

Mean Proportions of Critical Items Falsely Recalled and Falsely Recognized

\begin{tabular}{|c|c|c|c|c|c|c|c|c|c|}
\hline \multirow[b]{3}{*}{ Experiment } & \multirow[b]{3}{*}{ Condition } & \multicolumn{4}{|c|}{ False Recall } & \multicolumn{4}{|c|}{ False Recognition } \\
\hline & & \multicolumn{2}{|c|}{$\begin{array}{c}\text { Auditory } \\
\text { Presentation }\end{array}$} & \multicolumn{2}{|c|}{$\begin{array}{c}\text { Visual } \\
\text { Presentation }\end{array}$} & \multicolumn{2}{|c|}{$\begin{array}{c}\text { Auditory } \\
\text { Presentation }\end{array}$} & \multicolumn{2}{|c|}{$\begin{array}{c}\text { Visual } \\
\text { Presentation }\end{array}$} \\
\hline & & $M$ & $95 \% \mathrm{CI}$ & $M$ & $95 \% \mathrm{CI}$ & $M$ & $95 \% \mathrm{CI}$ & $M$ & $95 \% \mathrm{CI}$ \\
\hline \multirow[t]{2}{*}{1} & No recall; yes-no recognition test & & - & & - & .82 & $.78, .86$ & .81 & $.77, .85$ \\
\hline & Recall; yes-no recognition test & .48 & $.42, .54$ & .32 & $.28, .36$ & .86 & $.82, .90$ & .69 & $.63, .75$ \\
\hline \multirow[t]{2}{*}{2} & No recall; yes-no recognition test & & - & & - & .84 & $.78, .90$ & .81 & $.75, .87$ \\
\hline & Recall; yes-no recognition test & .35 & $.27, .43$ & .24 & $.20, .28$ & .80 & $.74, .86$ & .64 & $.56, .72$ \\
\hline \multirow[t]{2}{*}{3} & No recall; yes-no recognition test & & - & & - & .74 & $.66, .82$ & .81 & $.77, .85$ \\
\hline & No recall; remember-know recognition test & & - & & - & .75 & $.67, .83$ & .63 & $.55, .71$ \\
\hline
\end{tabular}

Note $-95 \% \mathrm{CI}=$ lower and upper bounds of the $95 \%$ confidence intervals. 
$\left[F(1,228)=12.21, M S_{\mathrm{e}}=.04, \eta_{\mathrm{p}}^{2}=.001\right]$ was qualified by a significant interaction of the two variables $[F(1,228)=$ 9.39, $\left.M S_{\mathrm{e}}=.04, \eta_{\mathrm{p}}^{2}=.04\right]$. The group that did have a recall test showed the standard reduction in false recognition following visual study, relative to following auditory study $\left[F(1,114)=19.07, M S_{\mathrm{e}}=.05, \eta_{\mathrm{p}}^{2}=.14\right]$. For the recall group, critical item false alarms were less likely following visual study presentation than following auditory study presentation. In contrast, there was no effect of study presentation modality on critical item false alarms for the group that completed a puzzle after each study list $(F<1)$.

False recognition given that the critical item was not recalled. ${ }^{3}$ Perhaps the reduction in false recognition for the visual study presentation condition when the recognition test was preceded by a recall test was due entirely to differences in recognition of words that were recalled. That is, false recognition of critical items in the auditory condition may have been higher because more of these items were falsely recalled in the auditory condition. To examine this possibility, we evaluated the probability of falsely recognizing a critical item, given that the item had not been recalled. One participant falsely recalled all of the critical items and was therefore excluded from this analysis.

The conditional analysis was done in the following way. For each participant, we determined which critical items had not been recalled. We then determined which of these nonrecalled critical items had been falsely called old on the recognition test. For each participant, we then divided the number of nonrecalled critical items that had been falsely recognized by the number of nonrecalled critical items. The proportion of nonrecalled critical items that had been falsely recognized was affected by study modality $\left[F(1,114)=16.89, M S_{\mathrm{e}}=.08, \eta_{\mathrm{p}}^{2}=.13\right]$. The mean proportion of critical items that had been falsely recognized but not falsely recalled was greater for participants in the auditory condition $(M=.79, S E M=.03)$ than in the visual condition $(M=.57, S E M=.04)$. The reduction on critical false alarms in recognition following visual study occurred for items that had not previously been recalled. This result replicates the results of the conditional analysis reported by Smith and Hunt (1998).

False recognition of weakly related and unrelated lures. We also evaluated the proportions of false alarms to the weakly related lures and the unrelated lures as a function of study modality for each group. As with the critical item false alarms, in the recall group significantly fewer false alarms occurred to weakly related lures following visual presentation $(M=.06, S E M=.01)$ than occurred following auditory presentation $(M=.17, S E M=.02)$ $\left[F(1,114)=19.05, M S_{\mathrm{e}}=.02, \eta_{\mathrm{p}}^{2}=.14\right]$. The effect of study modality on weakly related false alarms was not significant for the puzzle group $(M=.26, S E M=.02)$ $\left[F(1,114)=2.52, M S_{\mathrm{e}}=.04\right]$. Thus, as in previous research (e.g., Smith \& Hunt, 1998), false alarms to weakly related lures were not nearly as frequent as false alarms to critical items. Nonetheless, the weakly related lures produced the same pattern as the critical false alarms with respect to the effect of group and modality. Study modality did not significantly affect the proportion of false alarms to unrelated lures for either the recall group $[F(1,114)=$ $\left.1.25, M S_{\mathrm{e}}=.001\right]$ or the puzzle group $(F<1)$. False alarms to unrelated lures were rare, with a mean proportion of only .03 $($ SEM $=.004)$.

\section{Summary}

Visual study presentation reduced critical item false recall on written recall tests. Thus, the reduction in false memory following visual rather than auditory study, seen in 10 other experiments using recall tests (Cleary \& Greene, 2002; Gallo et al., 2001, Experiment 1; Kellogg, 2001; Smith \& Hunt, 1998; Smith et al., 2005), was replicated here. Results for the recognition test depended on whether the test was preceded by a recall test. When participants recalled the list words after each list presentation, visual study presentation significantly reduced critical item false alarms on the final recognition test. Visual study presentation did not influence critical false alarms when participants worked on a puzzle after each list instead of completing the recall test. The reduction in false recognition following visual study did not occur when recognition was not preceded by recall of the study list items.

\section{EXPERIMENT 2}

The goal of Experiment 2 was to replicate the results of Experiment 1 under different circumstances. In particular, the recall test in Experiment 2 occurred after all of the items had been presented, rather than after each list, as was the case in Experiment 1, and the condition in which no recall occurred did not include a distractor task between study and recognition testing. When a recall test preceded recognition, we expected to find a reduction in critical item false alarms on the recognition test following visual study. In light of the results of Experiment 1, we expected no reduction on false alarms to the critical items following visual study when recognition was not preceded by a recall test.

\section{Method}

\section{Design}

Participants were randomly assigned to either the recall or the no-recall group. Of the 47 participants in the recall condition, 23 were randomly assigned to the auditory condition and 24 to the visual condition. Half of the 50 participants in the no-recall group were randomly assigned to the auditory condition and half to the visual condition. All participants received the ordered recognition test. Thus, the experiment involved a 2 (recall condition: recall vs. no recall) $\times 2$ (modality: visual vs. auditory) design.

\section{Materials and Procedures}

The materials and procedures matched those of Experiment 1, with the following exceptions. In Experiment 1, we tested recall after each study list presentation. An alternative method that has been used in some previous studies is to present all of the study list items in a single list before testing recall. A reduction in false recall following visual study, as compared with auditory study, has been found using both a recall test after each set of associates and a single test following all associate lists (e.g., Smith \& Hunt, 1998; Smith et al., 2005). We used the single-list method in this experiment for the sake of generalizability.

The same six lists of 12 associates used in Experiment 1 were combined into a single 72-item study list, blocked by list, with each list of associates starting with the highest associate. Half of the par- 
ticipants heard the list, and half saw the list at study. Half of the participants completed a free recall test followed by the recognition test. The other half of the participants completed only the recognition test. In the latter case, we followed the procedures used in previous studies and presented instructions for the recognition test immediately after the end of the study list (e.g., Gallo et al., 2001; Pierce et al., 2005).

In the recall condition, participants were given a 2.5 -min written recall test following the study list presentation. Responses were written on a single sheet of paper. At the end of the recall test, the experimenter collected all recall sheets before participants read the instructions for the recognition test. Following the recall test for the recall group, or following the study list presentation for the no-recall group, participants received instructions for the yes-no recognition test. The test items were blocked in accord with the ordered test condition in Experiment 1.

\section{Results and Discussion}

\section{Recall}

In a mixed ANOVA of the proportion of items produced on the recall test with the repeated measure of item type (critical vs. studied) and the between-subjects measure of study presentation modality, neither the main effect of item type $(F<1)$ nor that of study modality $[F(1,45)=$ $\left.2.71, M S_{\mathrm{e}}=.03, p<.10\right]$ reached significance. However, the interaction of the two variables was significant $\left[F(1,45)=6.08, M S_{\mathrm{e}}=.02, \eta_{\mathrm{p}}^{2}=.12\right]$. Separate analyses were conducted for each item type in order to investigate the source of this interaction.

Correct recall. Correct recall was not influenced by study presentation modality $(F<1)$. The mean proportion of words correctly recalled was $.29(S E M=.02)$ in both the visual and auditory conditions. Given the longer list, it is not surprising that the level of recall was lower than it was in Experiment 1.

False recall. The proportion of critical items that were incorrectly produced on the recall test is shown in Table 1 as a function of modality. Visual presentation significantly reduced critical item false recall, relative to auditory presentation $\left[F(1,45)=5.14, M S_{\mathrm{e}}=.03, \eta_{\mathrm{p}}^{2}=.03\right]$. Thus, the reduction in critical false alarms following visual study relative to following auditory study on written recall tests was replicated in this experiment.

List access. When testing recall following a single long list, there is a possibility of differential list access. List access refers to recall of at least one item from a given associate list. In Experiment 1, where recall was tested immediately following each list, participants always recalled at least one item, making list access perfect; thus, we did not evaluate this dependent measure in Experiment 1. For Experiment 2, we calculated the proportion of the six lists for which at least one item was recalled, since this can potentially provide additional information about how visual study presentation reduces later false recall.

The proportion of lists accessed was not significantly affected by modality $(F<1)$. The mean proportion of lists from which at least one item was recalled was .83 (SEM $=$ $.04)$ following auditory study and $.79(S E M=.03)$ following visual study. The reduction in false recall associated with visual study presentation seen in this experiment was not a result of differential list access during the recall test and was more likely due to differences in access to critical items or in the ability to reject the highly associated critical items once an associate list had been accessed.

\section{Recognition}

We conducted a mixed ANOVA with the repeated measure of item type and the between-subjects factors of study modality and recall condition (recall vs. no recall). The significant main effect of item type $[F(1,93)=6.40$, $\left.M S_{\mathrm{e}}=.02, \eta_{\mathrm{p}}^{2}=.06\right]$ was qualified by a significant interaction between item type and study modality $[F(1,93)=$ $\left.8.75, M S_{\mathrm{e}}=.02, \eta_{\mathrm{p}}^{2}=.09\right]$. The main effect of recall condition $\left[F(1,93)=6.09, M S_{\mathrm{e}}=.04, \eta_{\mathrm{p}}^{2}=.06\right]$ was significant, and the interaction of recall condition and item type approached significance $\left[F(1,93)=3.73, M S_{\mathrm{e}}=.02, p<\right.$ $\left..06, \eta_{\mathrm{p}}^{2}=.04\right]$. No other effects or interactions reached significance (all $F_{\mathrm{S}}<2.26$, all $p \mathrm{~s}>.13$ ). We followed the omnibus test with separate analyses for each item type.

Correct recognition. The proportion of study list items correctly called old on the recognition test was not significantly affected by whether or not there was a recall test $\left[F(1,93)=1.18, M S_{\mathrm{e}}=.02\right]$. The main effect of study modality and the interaction were not significant $\left(F_{\mathrm{S}}<1\right)$. The mean proportion of studied items correctly identified as such was .80 following auditory study presentation and .81 following visual study presentation when the recognition test was preceded by a recall test and was .82 and .85 in the auditory and visual study conditions, respectively, when there was no prior recall test ( $S E M=.03$ in all cases).

False recognition. The proportion of critical item false alarms is shown in Table 1 . Both modality $[F(1,93)=5.84$, $\left.M S_{\mathrm{e}}=.04, \eta_{\mathrm{p}}^{2}=.06\right]$ and recall condition $[F(1,93)=8.05$, $\left.M S_{\mathrm{e}}=.04, \eta_{\mathrm{p}}^{2}=.08\right]$ reliably influenced false alarms to critical items. Although the interaction between modality and group did not quite reach reliable levels $[F(1,93)=$ $2.96, M S_{\mathrm{e}}=.04, p<.09, \eta_{\mathrm{p}}^{2}=.03$ ], planned comparisons were conducted in order to test the a priori prediction that false recognition would be reduced following visual study presentation as compared with auditory study presentation only when the recognition test was preceded by a recall test. In the case of the recall group, visual study presentation significantly reduced critical item false alarms, as compared with auditory study presentation $[F(1,45)=$ $\left.6.52, M S_{\mathrm{e}}=.05, \eta_{\mathrm{p}}^{2}=.13\right]$. In contrast, study presentation modality did not affect critical item false alarms in the group that did not have the recall test before the recognition test $(F<1)$. The pattern of results replicates the findings in Experiment 1: A reduction in false recognition for the visual study modality relative to the auditory study modality occurred only when the recognition test was preceded by a recall test. ${ }^{4}$

False recognition given that the critical item was not recalled. As in Experiment 1, we wanted to rule out the possibility that the reduction in false recognition associated with visual study presentation was entirely driven by differential recall of the critical items. Once again, there was a significant effect of study presentation modality on false recognition of critical items that had not been recalled $\left[F(1,45)=4.38, M S_{\mathrm{e}}=.08, \eta_{\mathrm{p}}^{2}=.09\right]$. Participants in the auditory condition falsely recognized a greater proportion of the critical items that had not been 
recalled $(M=.72, S E M=.06)$ than did those in the visual condition $(M=.55, S E M=.05)$.

False recognition of weakly related and unrelated lures. We examined the proportions of false alarms for the weakly related lures and the unrelated lures separately for each group. Modality did not significantly affect the proportion of false alarms to weakly related lures in either the recall group or the no-recall group $\left(F_{\mathbf{s}}<1\right)$. The mean proportion of false alarms to weakly related lures, collapsing across-modalities, was $13(S E M=.02)$ for the recall group and $.06(S E M=.01)$ for the no-recall group. This pattern of results differs from that of Experiment 1, in which we found a significant effect of modality on weakly related lures in the recall group. It is possible that the procedural changes account for this difference. The proportion of false alarms to unrelated lures was not affected by modality in either group $\left(F_{\mathrm{s}}<1\right)$. As in Experiment 1, there were few false alarms to unrelated lures. The mean proportion of unrelated false alarms was .04 $($ SEM $=.01)$.

\section{Summary}

The reduction in false recall following visual study presentation, relative to false recall following auditory study presentation, again was replicated, as was the subsequent reduction in false recognition of critical distractors when the recognition test followed a recall test. Unlike the previous demonstrations of a reduction in false recognition associated with visual study presentation following recall of individual lists of associates (Experiment 1; Cleary \& Greene, 2002; Gallo et al., 2001; Smith \& Hunt, 1998), the present data extend this finding on recognition to the case in which recognition follows a single recall test for all of the associate lists. Importantly, we also found that the visual study presentation did not reduce false recognition when the recognition test was not preceded by a recall test, replicating the results of Experiment 1.

\section{EXPERIMENT 3}

The findings from Experiments 1 and 2 are consistent with our motivating hypotheses. However, the literature does contain reports of reductions in false memory on recognition tests that were not preceded by recall tests (Gallo et al., 2001; Gallo \& Roediger, 2003; Pierce et al., 2005). As was mentioned in the introduction, the recognition tests used in the previous studies differed in an important way from the recognition tests used in Experiments 1 and 2 of the present study. In Experiments 1 and 2, we used yes-no recognition tests, but in the studies conducted by Gallo and his colleagues (Gallo et al., 2001; Gallo \& Roediger, 2003; Pierce et al., 2005), the recognition test required a remember-know or source judgment. Reaching a remember-know decision may require more extensive evaluation of memory than does a simple yes-no recognition decision. If so, it is possible that a remember-know judgment encourages the same sort of evaluative processing that occurs in free recall. On the basis of our premises, such processing can reduce or eliminate the beneficial effect that normally accrues to visual study processing.
In Experiment 3, we evaluated the prediction that inclusion of remember-know decisions on a recognition test would encourage additional processing of the quality of a memory, thereby leading to a reconstruction of additional contextual information. This in turn would lead to the reduction in false recognition following visual study, relative to auditory study, without a preceding recall test. In Experiment 3, we manipulated the type of recognition test: yes-no versus remember-know-guess-new (RKGN). Within each test type condition, half of the participants saw the study words, whereas the other half heard the study list words. We predicted that the visual study presentation would reduce false recognition, relative to auditory study presentation on the RKGN test but not on the yes-no test.

\section{Method}

\section{Participants and Design}

One hundred twenty-two participants were randomly assigned to one of four conditions formed by the between-subjects manipulation of study modality and recognition test type. Sixty-two participants (32 in the auditory study condition and 30 in the visual study condition) received the yes-no recognition test used in the first two experiments. Sixty participants ( 31 in the auditory condition and 29 in the visual condition) received RKGN instructions for the recognition memory test.

\section{Materials and Procedures}

The procedures for the study phase matched those of Experiment 2 . The study list presentation was followed by either a yes-no or an RKGN recognition test. The yes-no instructions matched those used in the previous two experiments: The participants were asked to respond by pressing the "Y" key for yes if the word was one from the study list or the "N" key for no if the word was not on the study list. In the RKGN condition, participants were asked to press the " $\mathrm{R}$ " key for remember if they could recollect details about the word's presentation, such as what the word looked or sounded like. The participants were asked to press the "K" key for know if they did not recollect specific details about the word but still believed that the word had been in the study list. The "G" for guess response was to be used if the participants did not recollect details and did not have a feeling of knowing that the word had been in the list but still felt that they recognized the word. Finally, participants pressed the "N" key for new if the word had not been in the list. The test stimuli matched the ordered recognition test used in Experiments 1 and 2. As in the previous experiments, in the yes-no condition, the reminder to press "Y" if the word had been in the list and " $N$ " if the word had not been in the list appeared below each test item. In the RKGN condition, the following reminders were shown with each test item: to press " $R$ " if they remembered that the word had been in the list, " $\mathrm{K}$ " if they knew that the word had been in the list, "G" if they guessed that the word was in the list, and " $\mathrm{N}$ " if the word was new.

\section{Results and Discussion}

We first analyzed the results for the two test conditions together. As in Experiment 2, in which only the yes-no recognition test was employed, study list items receiving a yes response were considered correct responses, and critical lures receiving a yes response were considered false recognition responses. In the RKGN test, both remember and know responses were combined to produce a single measure of correct recognition for studied items and false recognition for critical lures. This allowed direct comparison of the two recognition tests. Following discussion of 
this analysis, we will present the more fine-grained analysis for the RKGN test condition.

A mixed ANOVA including the repeated measure of item type (study list items vs. critical lure) and the between-subjects variables of study modality and recognition test type (yes-no vs. RKGN) showed significant main effects of item type $\left[F(1,118)=11.86, M S_{\mathrm{e}}=.02\right.$, $\left.\eta_{\mathrm{p}}^{2}=.09\right]$ and test type $\left[F(1,118)=4.47, M S_{\mathrm{e}}=.05\right.$, $\left.\eta_{\mathrm{p}}^{2}=.04\right]$. The interaction of study modality and test type was significant $\left[F(1,118)=6.09, M S_{\mathrm{e}}=.05, \eta_{\mathrm{p}}^{2}=\right.$ $.05]$ and there was a trend toward a significant interaction of item type and test type $\left[F(1,118)=2.84, M S_{\mathrm{e}}=\right.$ $\left..02, p<.10, \eta_{\mathrm{p}}^{2}=.02\right]$. The three-way interaction did not reach significance $\left[F(1,118)=2.45, M S_{\mathrm{e}}=.02, p>.12\right]$ and no other effects or interactions reached significance $(F \mathrm{~S}<1)$. We conducted separate analyses for correct and false recognition.

\section{Correct Recognition}

Neither main effect reached significance in the analysis of correct recognition involving the between-subjects factors of study presentation modality $(F<1)$ and recognition test type $\left[F(1,118)=1.35, M S_{\mathrm{e}}=.02\right]$. The two variables did not interact $\left[F(1,118)=2.64, M S_{\mathrm{e}}=.02\right]$. The mean proportion of study list items correctly identified as having been on the study list on the yes-no recognition test was $.79(S E M=.02)$ in the auditory condition and $.83(S E M=.02)$ in the visual condition. The mean proportion of study list items given either a remember or a know response on the RKGN recognition test was .80 (SEM = $.03)$ in the auditory condition and .76 $(S E M=.03)$ in the visual condition.

\section{False Recognition}

In an analysis of the proportion of critical item false alarms (Table 1), including both study modality and recognition test type as between-subjects factors, the effect of study presentation modality was not significant $(F<1)$. The main effect of recognition test type $[F(1,118)=5.20$, $\left.M S_{\mathrm{e}}=.05, \eta_{\mathrm{p}}^{2}=.04\right]$ was qualified by the significant interaction of the two variables $\left[F(1,118)=6.17, M S_{\mathrm{e}}=\right.$ $\left..05, \eta_{\mathrm{p}}^{2}=.05\right]$. In order to investigate this interaction, separate analyses were conducted for each test type. Modality of study presentation did not significantly affect performance on the yes-no recognition test $[F(1,60)=$ $\left.1.90, M S_{\mathrm{e}}=.04\right]$. This provides yet another replication of the findings from Experiments 1 and 2: Visual study presentation did not reduce false memories on a yes-no recognition test when that test was not preceded by a recall test. In contrast to the results for the yes-no test, but consistent with our predictions, critical item false alarms were significantly lower in the visual study condition than in the auditory study condition when the recognition test required an RKGN decision $\left[F(1,58)=4.26, M S_{\mathrm{e}}=.06\right.$, $\left.\eta_{\mathrm{p}}^{2}=.07\right]$.

\section{RKGN Response Selection}

We conducted additional analyses for the RKGN test condition to evaluate the effects of study modality on the selection of the four different response options (remember, know, guess, or new) first for critical items and then for study items (see Table 2).

Responses to critical items. For critical items, there was a significant interaction of study modality and response type $\left[F(3,174)=5.93, M S_{\mathrm{e}}=.07, \eta_{\mathrm{p}}^{2}=.09\right]$. Participants in the auditory study condition were significantly more likely than participants in the visual condition to give a remember response to critical items $[F(1,58)=12.09$, $\left.M S_{\mathrm{e}}=.08, \eta_{\mathrm{p}}^{2}=.17\right]$. The higher rate of know $[F(1,58)=$ $\left.3.39, M S_{\mathrm{e}}=.07, p<.08\right]$ and guess $[F(1,58)=3.05$, $\left.M S_{\mathrm{e}}=.03, p<.09\right]$ responses for visual study relative to auditory study only approached significance. The two groups did not differ with respect to the selection of new as a response for critical items $(F<1)$.

Response to studied items. In the analysis of response type selection for study list items, the interaction of response type and study modality was significant $\left[F(3,174)=3.19, M S_{\mathrm{e}}=.07, \eta_{\mathrm{p}}^{2}=.05\right]$. As with the critical lures, the proportion of studied items receiving a remember response was significantly greater following auditory study than following visual study $[F(1,58)=4.51$, $\left.M S_{\mathrm{e}}=.09, \eta_{\mathrm{p}}^{2}=.07\right]$. The effect of study modality on the rate of remember responses for studied items may initially seem surprising. Given that visual study is argued to have its effect through increased distinctiveness, one might expect that the visual study group would have higher rates of remember responses for the study list items. However, it is not that visual study increases the distinctiveness of the study items themselves but, rather, that visual study increases the discriminability of studied and nonstudied items. Therefore, one would not expect that visual study modality would increase the rate of remember responses. No other group differences reached significance $(F<$ $2.68, p>.10$, in all cases).

Table 2

Mean Proportions of Critical Items and Studied Items Given Remember, Know, Guess, or New Responses in Experiment 3

\begin{tabular}{|c|c|c|c|c|c|c|c|c|}
\hline \multirow{3}{*}{$\begin{array}{l}\text { Recognition Test } \\
\text { Response Type }\end{array}$} & \multicolumn{4}{|c|}{ Critical Items } & \multicolumn{4}{|c|}{ Studied Items } \\
\hline & \multicolumn{2}{|c|}{$\begin{array}{c}\text { Auditory } \\
\text { Presentation }\end{array}$} & \multicolumn{2}{|c|}{$\begin{array}{c}\text { Visual } \\
\text { Presentation }\end{array}$} & \multicolumn{2}{|c|}{$\begin{array}{c}\text { Auditory } \\
\text { Presentation }\end{array}$} & \multicolumn{2}{|c|}{$\begin{array}{c}\text { Visual } \\
\text { Presentation }\end{array}$} \\
\hline & $M$ & $95 \% \mathrm{CI}$ & $M$ & $95 \% \mathrm{CI}$ & $M$ & $95 \% \mathrm{CI}$ & $M$ & $95 \% \mathrm{CI}$ \\
\hline Remember & .55 & $.45, .65$ & .30 & $.22, .38$ & .5 & $.44, .64$ & .37 & $.25, .49$ \\
\hline Know & .20 & $.12, .28$ & .33 & $.21, .45$ & .26 & $.16, .36$ & .38 & $.26, .50$ \\
\hline Guess & .11 & $.07, .15$ & .18 & $.10, .26$ & .07 & $.05, .09$ & .11 & $.05, .17$ \\
\hline New & .14 & $.06, .22$ & .19 & $.11, .27$ & .13 & $.07, .19$ & .14 & $.10, .18$ \\
\hline
\end{tabular}

Note- $95 \% \mathrm{CI}=$ lower and upper bounds of the $95 \%$ confidence intervals. 


\section{GENERAL DISCUSSION}

Previous research using the DRM paradigm has shown that false memory is reduced following visual study relative to auditory study (Cleary \& Greene, 2002; Gallo et al., 2001; Gallo \& Roediger, 2003; Kellogg, 2001; Pierce et al., 2005; Smith \& Hunt, 1998; Smith et al., 2005). Although the reduction in false memories following visual study presentation appears to be reliable in our experiments, some experiments have reported relatively small reduction in false recognition following visual study relative to auditory study presentation (e.g., Gallo et al., 2001; Gallo \& Roediger, 2003), a numerical increase in false recognition following visual study relative to auditory study (Maylor \& Mo, 1999), or no effect of study modality on false memory (McKone \& Murphy, 2000). The goal of our research was to investigate potential factors that moderate the effects of visual study on false recognition.

The results of the experiments reported here include four important findings about the effects of study presentation on false memories. First, visual study reduced critical false alarms, relative to auditory study, on a yes-no recognition test only if a recall test preceded the recognition test. Second, the reduction in false recognition associated with visual study presentation occurred for nonrecalled critical items. The latter finding is important, because it indicates that the reduction in false recognition following visual study presentation is not due to recall of fewer critical items following visual study but, rather, that the prior recall test influences processing during recognition. Third, the order of the recognition test items did not affect whether study presentation reduced false recognition. Finally, visual study presentation can reduce false memories on a recognition test that is not preceded by a recall test when the recognition test employs RKGN recognition test instructions.

\section{Theoretical Perspectives}

Our explanation for these effects draws on the distinctiveness explanation of the influence of study modality on false memory offered by Smith and Hunt (1998). This explanation begins with the assumption that relational processing can influence activation of an item at study and/or at test. Although the critical item and studied items share relational information in the form of semantic association, the critical item does not share the perceptual information with the studied items. The perceptual processing of studied items can serve as a basis for discriminating those items from critical nonstudied items. Furthermore, auditory processing is more like the thought corresponding to the activated critical item than is visual processing. Hence, visual processing increases the chance that critical items will be distinguished from studied items. The discrimination could be accomplished by either of two mechanisms, which are not mutually exclusive.

Hege and Dodson (2004) proposed that distinctive processing of studied items reduces false alarms by reducing access to the critical lures at test. In this context, distinctive processing is any operation shared by all of the studied items but not by the critical lure, and as such, this distinctive processing is relational information for the studied items. This relational information serves in part to define the episode, in such a way that when memory is later requested for that episode, items that did not share that processing at study are unlikely to come to mind. For example, suppose that I am asked to describe the view of the coast that I experienced on my recent vacation. I am unlikely to report whatever conversation may have been occurring at the time that I experienced the view, because the conversation did not share the relational processing cued by the request to recall the view. In other words, because the critical item did not share relational information that was shared by the studied items, the critical item may be less likely to come to mind during subsequent recall. Hege and Dodson provided evidence in support of this explanation for why pictorial presentation reduces false alarms to critical lures in the DRM paradigm.

A second mechanism through which distinctive study processing can operate is an output-monitoring process. The idea begins in the same way as the limited accessibility explanation: The studied items are processed differently than the lures. But rather than reducing accessibility to the lures, the distinctive processing is used to discriminate presented from nonpresented items at output. Schacter and his colleagues (e.g., Schacter, Israel, \& Racine, 1999) have championed this view under the rubric of the distinctiveness heuristic, and most researchers in the area agree that this process does occur (Arndt \& Reder, 2003; Hege \& Dodson, 2004; Roediger, Watson, McDermott, \& Gallo, 2001; Smith \& Hunt, 1998).

Again, these two mechanisms are not mutually exclusive, and both are reasonable explanations of the effect of distinctive processing on rejection of distractors. In the experiments reported here, the monitoring account seems best suited to some of the data. In particular, the difference in the effect of visual study presentation on false memory as a function of the type of recognition test does not conform to what might be expected from the accessibility account. There is little reason to suppose that the difference between a remember-know test and a yes-no test would affect the accessibility of the critical lure. On the other hand, it is quite reasonable that the remember-know judgment would intensify the monitoring process.

\section{Yes-No Versus Remember-Know Recognition Tests}

Our idea also explains the results of three experiments that do show a reduction in false memory following visual study even though no recall test preceded the recognition test. The data in question are from Gallo et al. (2001), Gallo and Roediger (2003), and Pierce et al. (2005), showing a reduction in false recognition following visual study relative to auditory study in the absence of a prior recall test. Unlike the recognition tests of Cleary and Greene (2002) and Smith and Hunt (1998) that required a simple yes-no response, these experiments by Gallo and his associates (Gallo et al., 2001; Gallo \& Roediger, 2003; Pierce et al., 2005) used recognition tests that required both a yes-no response and a remember-know-guess or source judgment. We suggest that the remember-know judgment task in recognition encourages more scrutiny of memory 
than does the yes-no judgment and that the task results in consideration of differences among items much as does the process we described for recall. The results of Experiment 3 , which directly contrasted a yes-no recognition test and a remember-know recognition test, are consistent with this explanation.

Although the results of this study indicated that remember-know tests encourage additional scrutiny of potential responses relative to yes-no recognition tests, the results suggest that this additional processing may not be equivalent to the response evaluation processes encouraged by free recall tests. Specifically, the reduction in critical false alarms in the visual condition relative to the auditory condition was at least numerically smaller in the remember-know test of Experiment 3 (.12), relative to the reduction in critical false alarms when the recognition test followed a recall test in Experiments 1 and 2 (.17 and .16 , respectively).

\section{Summary}

In conclusion, the results presented here point to limitations on the influence of study modality on false recognition in the DRM paradigm. Specifically, visual study reduces false recognition when the recognition follows a recall test or when the recognition test encourages more careful scrutiny of the nature of one's memory. We attribute the reduction in false memory to distinctive processing of the critical item relative to the list items on the basis of the assumption that auditory perception is more similar to thought than is visual perception (Smith \& Hunt, 1998). The distinctive study processing can affect retrieval in one of two ways, which are not mutually exclusive. The distinctively processed item may not come to mind during the test (Hege \& Dodson, 2004) because the dimension of difference between the critical item and the studied items is an important component of the relational information shared by the studied items. Alternatively, the distinctive study processing may allow more accurate monitoring of items that do come to mind at test by enhancing the discriminability of studied and nonstudied items. The data presented here seem to favor the latter alternative in that the manipulations that eliminated the modality effect occurred after the study experience. If visual processing prevented the critical items from coming to mind, the probability of falsely recognizing those items should be reduced regardless of the type of processing required by the test.

The outcome of this research contributes to the general question of how distinctive processing influences memory. In the case of false recognition, distinctive processing seems to affect the caliber of output monitoring. In addition, the research clearly suggests that the monitoring processes are affected both by the demands of the test and by prior testing experience. Similar conclusions have been reached following studies of reality monitoring (e.g., Marsh \& Hicks, 1998). Thus, distinctive processing certainly contributes to memory accuracy, but that contribution, when it arises through effects on output monitoring, will be modulated by other influences on the quality of the monitoring process.

\section{AUTHOR NOTE}

This research was supported in part by Grant R15 MH067582 from the National Institute of Mental Health (to R.R.H.), and by Individual National Research Service Award F32 AG20021 from the National Institute on Aging (to R.E.S.). We thank Jessica Scorso, Elizabeth Cedillo, and Jeremiah van Winkle for assistance with data collection. Correspondence concerning this article should be addressed to R. E. Smith, Department of Psychology, University of Texas at San Antonio, San Antonio, TX 78249 (e-mail: rebekah.smith@utsa.edu).

\section{REFERENCES}

ARndt, J., \& REDER, L. M. (2003). The effect of distinctive visual information on false recognition. Journal of Memory \& Language, 48 , $1-15$.

Cleary, A. M., \& Greene, R. L. (2002). Paradoxical effects of presentation modality on false memory. Memory, 10, 55-61.

DEESE, J. (1959). On the prediction of occurrence of particular verbal intrusions in immediate recall. Journal of Experimental Psychology, $\mathbf{5 8}, 17-22$.

Gallo, D. A. (2006). Associative illusions of memory: False memory research in DRM and related tasks. New York: Psychology Press.

Gallo, D. A., McDermott, K. B., Percer, J. M., \& Roediger, H. L., III (2001). Modality effects in false recall and false recognition. Journal of Experimental Psychology: Learning, Memory, \& Cognition, 27, 339-353.

Gallo, D. A., \& Roediger, H. L., III (2002). Variability among word lists in eliciting memory illusions: Evidence for associative activation and monitoring. Journal of Memory \& Language, 47, 469-497.

Gallo, D. A., \& Roediger, H. L., III (2003). The effects of associations and aging on illusory recollections. Memory \& Cognition, 31, 1036-1044.

Hege, A. C. G., \& Dodson, C. S. (2004). Why distinctive information reduces false memories: Evidence for both impoverished relational encoding and distinctiveness heuristic accounts. Journal of Experimental Psychology: Learning, Memory, \& Cognition, 30, 787-795.

Hicks, J. L., \& MARSH, R. L. (2001). False recognition occurs more frequently during source identification than during old-new recognition. Journal of Experimental Psychology: Learning, Memory, \& Cognition, 27, 375-383.

Hunt, R. R. (2003). Two contributions of distinctive processing to accurate memory. Journal of Memory \& Language, 48, 811-825.

Hunt, R. R., \& Einstein, G. O. (1981). Relational and item-specific information in memory. Journal of Verbal Learning \& Verbal Behavior, 20, 497-514.

Hunt, R. R., \& MCDAniel, M. A. (1993). The enigma of organization and distinctiveness. Journal of Memory \& Language, 32, 421-445.

Hunt, R. R., \& Worthen, J. B. (Eds.) (2006). Distinctiveness and memory. New York: Oxford University Press.

Johnson, M. K., Nolde, S. F., \& De Leonardis, D. M. (1996). Emotional focus and source monitoring. Journal of Memory \& Language, 35, 135-156.

KellogG, R. T. (2001). Presentation modality and mode of recall in verbal false memory. Journal of Experimental Psychology: Learning, Memory, \& Cognition, 27, 913-919.

Marsh, R. L., \& Hicks, J. L. (1998). Test formats change sourcemonitoring decision processes. Journal of Experimental Psychology: Learning, Memory, \& Cognition, 24, 1137-1151.

Mather, M., Henkel, L. A., \& Johnson, M. K. (1997). Evaluating characteristics of false memories: Remember/know judgments and memory characteristics questionnaire compared. Memory \& Cognition, 25, 826-837.

MAYLOR, E. A., \& Mo, A. (1999). Effects of study-test modality on false recognition. British Journal of Psychology, 90, 477-493.

MCKone, E., \& MurPhy, B. (2000). Implicit false memory: Effects of modality and multiple study presentations on long-lived semantic priming. Journal of Memory \& Language, 43, 89-109.

Payne, D. G., Elie, C. J., Blackwell, J. M., \& Neuschatz, J. S. (1996). Memory illusions: Recalling, recognizing, and recollecting events that never occurred. Journal of Memory \& Language, 35, 261-285.

Pierce, B. H., Gallo, D. A., Weiss, J. A., \& Schacter, D. L. (2005). 
The modality effect in false recognition: Evidence for test-based monitoring. Memory \& Cognition, 33, 1407-1413.

READ, J. D. (1996). From a passing thought to a false memory in 2 minutes: Confusing real and illusory events. Psychonomic Bulletin \& Review, 3, 105-111.

Roediger, H. L., III, \& McDermott, K. B. (1995). Creating false memories: Remembering words not presented in lists. Journal of Experimental Psychology: Learning, Memory, \& Cognition, 21, 803-814.

Roediger, H. L., III, Watson, J. M., McDermott, K. B., \& Gallo, D. A. (2001). Factors that determine false recall: A multiple regression analysis. Psychonomic Bulletin \& Review, 8, 385-407.

Schacter, D. L., Israel, L., \& Racine, C. (1999). Suppressing false recognition in younger and older adults: The distinctiveness heuristic. Journal of Memory \& Language, 40, 1-24.

Sмiтh, R. E., \& Hunt, R. R. (1998). Presentation modality affects false memory. Psychonomic Bulletin \& Review, 5, 710-715.

Smith, R. E., Lozito, J. P., \& Bayen, U. J. (2005). Adult age differences in distinctive processing: The modality effect in false recall. Psychology \& Aging, 20, 486-492.

\section{NOTES}

1. A reviewer suggested that existing empirical evidence regarding source judgments argues against the proposal that study modality presentation information does not serve as a source of shared relational information for the critical items. The reviewer did not cite a particular study, but at least two such studies fit the reviewer's general description (Gallo et al., 2001; Mather, Henkel, \& Johnson, 1997). Namely, when participants are asked to identify the source (e.g., Was the item presented by a female voice or a male voice? Was the item presented visually or auditorily at study?) during a final visual recognition test, participants are more likely to select the source corresponding to the associate study list items. In other words, if the list of associates corresponding to the critical item SWEET was presented in a female voice, participants were more likely to select this as the source for the falsely recognized critical item. It may in fact be, as the reviewer suggested, that these results suggest that study modality information is a source of "shared relational information" that serves as a cue for the nonpresented critical item. However, the evidence from source-monitoring studies can be interpreted in an alternative fashion. The critical item may be falsely recognized purely on the basis of shared relational information in the form of the shared semantic relationship between the critical item and the study list items. The fact that participants attribute the critical item to the same source as the associated study list items does not necessarily mean that the study modality information was serving as a cue for critical item false recognition. Instead, the semantic similarity between the critical item and associated study list items could serve as the basis for the participant to attribute the critical item to the same source on the basis of their memory for the source of the semantically related study list items. (See Gallo, 2006, for additional discussion of related issues.)

2. A similar proposal was made by Gallo and Roediger (2002) to explain different effects of study presentation rate on recognition in two experiments. Study presentation rate influenced false recognition when recall was tested before the recognition test (Gallo \& Roediger, 2002, Experiment 2), but false recognition was not influenced by study presentation rate when the recognition test was not preceded by a recall test (Gallo \& Roediger, 2002, Experiment 3). Gallo and Roediger (2002) suggested that recall tests may be more sensitive to monitoring processes than are recognition tests. Furthermore, they proposed that when a recall test precedes a recognition test, the increased discriminability of study and critical items may carry over to the recognition test.

3. We also analyzed the probability of calling a critical item old on the recognition test given that it had been falsely recalled. Eleven participants in Experiment 1 and 6 in Experiment 2 did not falsely recall any of the critical items and were therefore excluded from these analyses. Study presentation modality did not affect the probability of falsely recognizing an item once that item had been recognized in either Experiment 1 $(F<1)$ or Experiment $2\left[F(1,39)=1.05, M S_{\mathrm{e}}=.002\right]$. The mean proportion of falsely recalled critical items that were also falsely recognized was $.99(S E M=.01)$ in both experiments. In other words, the probability of falsely recognizing a critical item once that item had been falsely recalled was at ceiling.

4. During the review process, a reviewer proposed that perhaps the preceding recall test reduces false recognition generally and in this way contributes to the reduction in false recognition following visual study for the recall condition. This can be evaluated by examining false recognition in the auditory conditions. If the preceding recall test has a suppression effect on false recognition, the auditory condition should also show a reduction. In Experiment 1, false recognition was in fact numerically higher in the recall condition than in the puzzle condition, but this difference was not significant $\left[F(1,114)=2.07, M S_{\mathrm{e}}=.03\right]$. The difference in false recognition for the recall and no-recall conditions following auditory study was also not significant in Experiment $2(F<1)$. In contrast, the recall group had significantly lower false recognition than the no-recall group following visual study in Experiment $1[F(1,114)=$ $\left.7.56, M S_{\mathrm{e}}=.05, \eta_{\mathrm{p}}^{2}=.06\right]$ and Experiment $2\left[F(1,47)=9.01, M S_{\mathrm{e}}=\right.$ $\left..04, \eta_{\mathrm{p}}^{2}=.16\right]$. It does not appear that the preceding recall test generally suppresses false recognition. Instead, it appears that the preceding recall test facilitates reduction of false recognition following visual study.

(Manuscript received April 19, 2006; revision accepted for publication July 28, 2008.) 